\title{
The Price of Anarchy in Network Creation Games Is (Mostly) Constant
}

\section{Journal Article}

\section{Author(s):}

Mihalák, Matúš; Schlegel, Jan Christoph

Publication date:

2013

Permanent link:

https://doi.org/10.3929/ethz-b-000423113

\section{Rights / license:}

In Copyright - Non-Commercial Use Permitted

Originally published in:

Theory of Computing Systems 53, https://doi.org/10.1007/s00224-013-9459-y 


\title{
The Price of Anarchy in Network Creation Games Is (Mostly) Constant
}

\author{
Matúš Mihalák · Jan Christoph Schlegel
}

Published online: 17 March 2013

(C) Springer Science+Business Media New York 2013

\begin{abstract}
We study the price of anarchy and the structure of equilibria in network creation games. A network creation game is played by $n$ players $\{1,2, \ldots, n\}$, each identified with a vertex of a graph (network), where the strategy of player $i, i=$ $1, \ldots, n$, is to build some edges adjacent to $i$. The cost of building an edge is $\alpha>0$, a fixed parameter of the game. The goal of every player is to minimize its creation cost plus its usage cost. The creation cost of player $i$ is $\alpha$ times the number of built edges. In the SUMGAME variant, the usage cost of player $i$ is the sum of distances from $i$ to every node of the resulting graph. In the MAXGAME variant, the usage cost is the eccentricity of $i$ in the resulting graph of the game. In this paper we improve previously known bounds on the price of anarchy of the game (of both variants) for various ranges of $\alpha$, and give new insights into the structure of equilibria for various values of $\alpha$. The two main results of the paper show that for $\alpha>273 \cdot n$ all equilibria in SUMGAME are trees and thus the price of anarchy is constant, and that for $\alpha>129$ all equilibria in MAXGAME are trees and the price of anarchy is constant. For SuMGaME this answers (almost completely) one of the fundamental open problems in the field - is price of anarchy of the network creation game constant for all values of $\alpha$ ? - in an affirmative way, up to a tiny range of $\alpha$.
\end{abstract}

Keywords Network creation games · Nash equilibrium · Price of anarchy

A preliminary version of this article appeared in the proceedings of the Third International Symposium on Algorithmic Game Theory (SAGT), 2010.

Most of the work on the paper has been done while Jan Christoph Schlegel was a student at ETH Zurich.

M. Mihalák (凶)

Institute of Theoretical Computer Science, ETH Zurich, Zurich, Switzerland

e-mail: matus.mihalak@inf.ethz.ch

J.C. Schlegel

Faculty of Business and Economics, University of Lausanne, Lausanne, Switzerland

e-mail: janchristoph.schlegel@unil.ch 


\section{Introduction}

Network creation game, as defined and introduced by Fabrikant et al. [8], is a game that models the process of building large autonomous computer and communication networks, such as the Internet. In this game, as in the reality, these networks are built and maintained by entities (players in the game-theoretic jargon) that pursue their own goals that may be different from the goals of other players- the players do not necessarily cooperate, they are selfish (we leave the real-world meaning of this on an intuitive level). Network creation games, and its superclass network formation games, is a well-studied and well-known research topic which is covered by many lectures and courses on algorithmic game theory and related subjects (see [18] for an overview of network formation games).

Network creation game is a strategic game with $n$ players where each player is identified with a vertex (of a to be built graph/network). Every player $i$ has to decide what edges incident to $i$ the player creates (or buys, or builds). Building one edge costs the player $\alpha>0$, which is a fixed parameter of the game. The edges that the players buy form a graph (network) which is the result of the game. The players pursue two incompatible goals: pay as little as possible (minimize the creation cost), and have a good connection to other nodes of the network (maximize the usage utility). The usage utility of player $i$ has been originally expressed as the following usage cost: the sum of distances to all other players in the resulting network [8] (where naturally players want to minimize this sum). Recently, the game where the usage cost of player $i$ is expressed as the maximum distance of $i$ to any node of $G$ has been studied [6]. In this paper we consider both variants.

The central question that motivated the study of network creation games is: what do we lose in terms of quality of a network, if the communication network is built autonomously by selfish agents, as opposed to a communication network that is centrally planned and built? The price of anarchy of a game is a way to express this in that one compares the cost of a worst Nash equilibrium ${ }^{1}$ (worst in the sense of the cost of the network) with the cost of an optimum network - the ratio of these two values is the price of anarchy of the game. Price of anarchy resembles in many aspects the approximation ratio used for quantifying quality of algorithms for optimization problems.

The challenge of computing the price of anarchy of any game is to find (compute) a maximum cost Nash equilibrium, and a minimum cost "configuration"- two optimization problems that may be computationally hard/challenging. Often one is not interested to know the exact value of the price of anarchy but only an approximation of it. For the network creation games, the quest has always been to identify the cases where the price of anarchy is constant, and for the other cases to get as good an asymptotic bound on the price of anarchy as possible.

\footnotetext{
${ }^{1}$ Nash equilibrium of the network creation game is a set of players' strategies, one for each player, such that no player can unilaterally change its strategy (i.e., buy a different set of edges) and improve her/his cost.
} 


\subsection{Definition of the Game and Related Concepts}

Let $G=(V, E)$ be an undirected graph (and we shall only consider undirected graphs in the paper). For $u, v \in V$ we denote by $d_{G}(u, v)$ the length of a shortest $u$-v-path in $G$, and by $D_{G}(v)$ the eccentricity of the vertex $v$, i.e., the maximum distance between $v$ and any other vertex of $G$. If $G$ is not connected we define $d_{G}(u, v):=\infty$. We denote the degree of vertex $v \in V$ in $G$ by $\operatorname{deg}_{G}(v)$. The $a v$ erage degree of $G$ is $\operatorname{deg}(G):=\frac{1}{|V|} \sum_{v \in V} \operatorname{deg}_{G}(v)=\frac{2|E|}{|V|}$. We sometimes omit the index $G$ and write simply $d(u, v), D(v)$, or $\operatorname{deg}(v)$ if the underlying graph $G$ is clear from the context. For $k \in \mathbb{N}$ we define the $k$-neighborhood of a vertex $v \in V$ as the set $N_{k}(v):=\{w \in V: d(v, w) \leq k\}$ (observe that $v$ belongs to $N_{k}$ ), and the boundary of the $k$-neighborhood as the set $N_{k}^{=}(v):=\{w \in V: d(v, w)=k\}$. Furthermore we define the set of all eccentric vertices of $v$ by $\mathcal{E}(v):=N_{\bar{D}(v)}^{\bar{N}}(v)$. We denote the diameter of $G$ by $\operatorname{diam}(G)$, and the radius of $G$ by $\operatorname{rad}(G)$. Recall that $\operatorname{diam}(G)=\max _{u, v \in V} d(u, v)$ and $\operatorname{rad}(G)=\min _{v} D(v)$. A central vertex is a vertex $v$ for which $D(v)=\operatorname{rad}(G)$. Graph $G$ is a star if it is a tree and all edges of $G$ are incident to one vertex. Recall that a biconnected graph is a graph that does not contain a cut vertex, i.e., a vertex whose removal makes the graph disconnected. Recall furthermore that a biconnected component (or a block) of a graph $G$ is a maximal biconnected subgraph of $G$.

We consider $n$ players $N=\{1, \ldots, n\}$ in our setting. Let $\alpha>0$ be a real number which we shall call the edge price. The set of strategies of player $i \in N$ is the set $S_{i}=$ $2^{N \backslash\{i\}}$ (i.e., $S_{i}$ contains all subsets of the set $N \backslash\{i\}$ ). A strategy $s_{i} \in S_{i}$ corresponds to a set of players to which $i$ buys (or builds) an edge. We define $S:=S_{1} \times S_{2} \times \cdots \times S_{n}$ and call the elements of $S$ the strategy profiles. For every strategy profile $s \in S$ we define the graph $G(s):=\left(N, \bigcup_{i=1}^{n} \bigcup_{j \in s_{i}}\{\{i, j\}\}\right)$, and a cost function $c_{i}(s)$ for every player $i$ (to be specified later). The triple $(N, S, c)$, where $c: S \longrightarrow \mathbb{R}^{n}$ is given by $c(s):=\left(c_{1}(s), \ldots c_{n}(s)\right)$, naturally defines a non-cooperative $n$-player strategic game. Depending on the form of $c_{i}(\cdot)$ we distinguish two games. Sum-Unilateral Network Creation Game, or shortly SuMGAmE, is the game given by $(N, S, c)$ where for $s \in S, i \in N$,

$$
c_{i}(s)=\alpha \cdot\left|s_{i}\right|+\sum_{j=1, \ldots, n} d_{G(s)}(i, j) .
$$

Max-Unilateral Network Creation Game, or shortly MAXGAME, is the game given by $(N, S, c)$ where for $s \in S, i \in N$,

$$
c_{i}(s)=\alpha \cdot\left|s_{i}\right|+\max _{j=1, \ldots, n} d_{G(s)}(i, j) .
$$

We call the term $\alpha \cdot\left|s_{i}\right|$ in both cost functions (for SumGame and MAXGAME) the creation cost, and the term $\sum_{j=1, \ldots, n} d_{G(s)}(i, j)$ or $\max _{j=1, \ldots, n} d_{G(s)}(i, j)$ in the respective cost function the usage cost of player $i$. A Nash equilibrium (NE for short) of the game $(N, S, c)$ is a strategy-profile $s \in S$ such that for every player $i \in N$ and every strategy $\tilde{s_{i}} \in S_{i}$ we have $c_{i}(s) \leq c_{i}\left(s_{1}, \ldots, s_{i-1}, \tilde{s_{i}}, s_{i+1}, \ldots, s_{n}\right)$, i.e. no player can lower her cost by changing her strategy when all other players keep their strategies unchanged. Observe therefore that for every finite $\alpha$ every Nash equilibrium 
is a connected graph, and in every Nash equilibrium any edge is bought by at most one player. If $s \in S$ is a Nash equilibrium of the game $(N, S, c)$, we call $G(s)$ an equilibrium graph or sometimes a stable graph. The social cost $C$ of a strategyprofile $s \in S$ is defined, for the respective cost function of player $i$, as the sum of the individual costs of the players under this strategy-profile, i.e.:

$$
C(s)=\sum_{i=1}^{n} c_{i}(s)= \begin{cases}\alpha \cdot \sum_{i=1}^{n}\left|s_{i}\right|+\sum_{i=1}^{n} \sum_{j=1}^{n} d_{G(s)}(i, j) & \text { in SumGame, } \\ \alpha \cdot \sum_{i=1}^{n}\left|s_{i}\right|+\sum_{i=1}^{n} \max _{j=1, \ldots, n} d_{G(s)}(i, j) & \text { in MAXGAME. }\end{cases}
$$

Since for every graph $G=(V, E)$ on $n$ vertices there is a strategy-profile inducing this graph, the social cost function generalizes for any graph $G$ on $n$ vertices:

$$
\begin{aligned}
& C(G)=\alpha \cdot|E|+\sum_{v \in V} D_{G}(v), \quad \text { or } \\
& C(G)=\alpha \cdot|E|+\sum_{v \in V} \sum_{w \in V} d_{G}(v, w)
\end{aligned}
$$

for the respective cost function. We call a graph $G_{\mathrm{OPT}}$ minimizing the respective social cost function a social optimum. The price of anarchy (PoA for short) of a game (SUMGAME or MAXGAME) is defined as

$$
\max _{s \in S ; s \text { is NE }} \frac{C(G(s))}{C\left(G_{\mathrm{OPT}}\right)} .
$$

\subsection{Related Work}

Networks have been an important research topic in the economical and social sciences, as networks naturally model relationships between interacting entities. As such, a link between two entities is usually created upon mutual consensus ("if entity $A$ knows entity $B$ then entity $B$ knows entity $A$ " is a common assumption). For an overview of economical and social studies from this perspective we refer to the book by Jackson [10] and to the references therein. Strategic network formation in this framework has been studied with tools from cooperative game theory. The tradeoff between efficiency and stability for these kind of networks has been studied by Jackson and Wolinski [11].

We study networks where links can be created unilaterally (i.e., without an explicit agreement of both players at the ends of the respective edges) and where the payoff of the players reflects the cost for building the edges as well as the quality of the resulting network in terms of the players' distances in the network. The first game of this nature studied in the literature is SUMGAME.

Fabrikant et al. introduced SUMGAME in [8]. They proved an upper bound $O(\sqrt{\alpha})$ on price of anarchy (by showing that price of anarchy is bounded by the diameter of the equilibrium graph), and showed that every Nash equilibrium which is a tree has constant price of anarchy; we will use this result later on. Albers et al. [1] showed that price of anarchy is constant for $\alpha=O(\sqrt{n})$ (this was also independently and earlier discovered by Lin [15]) and for $\alpha \geq 12 n \lg n$. The latter result is achieved by 
showing that for $\alpha \geq 12 n \lg n$ all Nash equilibria are trees. Albers et al. also present a general upper bound $15\left(1+\left(\min \left\{\alpha^{2} / n, n^{2} / \alpha\right\}\right)^{1 / 3}\right)$ on price of anarchy for all $\alpha$, which shows that price of anarchy is $O\left(n^{1 / 3}\right)$ for all $\alpha$. Demaine et al. [6] show that price of anarchy is constant already for $\alpha=O\left(n^{1-\varepsilon}\right)$ for any fixed $\varepsilon \geq 1 / \lg n$, and show the general bound $2^{O(\sqrt{\lg n})}$ on price of anarchy for all $\alpha$.

Demaine et al. [6] introduced MAXGAME as a natural variant of network creation games, and showed that price of anarchy of MAXGAME is at most 2 for $\alpha \geq n$, $O\left(\min \left\{4^{\sqrt{\lg n}},(n / \alpha)^{1 / 3}\right\}\right)$ for $2 \sqrt{\lg n} \leq \alpha \leq n$, and $O\left(n^{2 / \alpha}\right)$ for $\alpha<2 \sqrt{\lg n}$.

An inherent modeling weakness of network creation games and of the solution concept of Nash equilibria is the underlying computational intractability. This was already pointed out by Fabrikant et al. [8]: it can be shown that in SUMGAME, computing a best response ${ }^{2}$ of a player is NP-hard. Recently, Alon et al. [2] proposed to consider a variant of network creation games along with a simpler and weaker concept of equilibrium for which a best response of a player can be computed in polynomial time. In this variant, called a basic network creation game, the players do not buy edges (hence, there is no edge price $\alpha$ ), and one does not consider strategy profiles of bought edges inducing a graph which may be in Nash equilibrium. Instead, Alon et al. introduce the concept of a swap equilibrium: A given graph is said to be in (swap) equilibrium if no player (a vertex) can decrease its usage cost by deleting any adjacent edge and creating a new adjacent edge (in their jargon, no player can swap an existing edge and improve). A drawback of the basic network creation games is that the results do not generalize to the original network creation games (consider, for example, a path on four vertices $1,2,3,4$, where vertex 2 buys the edge to vertex 1 and vertex 3 buys an edge to vertex 4 ; this is a Nash equilibrium, but not a swap equilibrium).

A natural modification of the concept, called the asymmetric swap equilibrium, generalizes Nash equilibria of the original network creation games [16]: a graph in which every edge is owned by one of its endpoints is said to be in asymmetric swap equilibrium if no player can swap its own edge and improve its usage cost. It follows that every Nash equilibrium in the (original) network creation game directly translates into an asymmetric swap equilibrium. Therefore, any structural properties on any asymmetric swap equilibria immediately hold for Nash equilibria of network creation games; it has been proven that any asymmetric swap equilibrium has at most one 2-edge connected component [16].

Another modification of the concept of swap equilibria comes from Lenzner [14] who defines a greedy equilibrium of a network creation game as a strategy profile in which no player can improve its cost by swapping an edge, or by deleting an existing edge, or by buying a new edge. In its paper [14], Lenzner studies how well greedy equilibria approximate Nash equilibria of the network creation games. Obviously, every Nash equilibrium is a greedy equilibrium, which in turn is asymmetric swap equilibrium.

The computational tractability of the new equilibria concepts allows the players to compute their best responses. This automatically invites for a study of a dynamic

\footnotetext{
${ }^{2} \mathrm{~A}$ best response of a player $i$ against given strategies of the other players is a strategy of player $i$ that minimizes the player $i$ 's cost, assuming the other players play the given (fixed) strategies.
} 
Table 1 Comparison of the previously known bounds for the price of anarchy in MAXGAME (due to [6]) and the bounds proved in this paper

\begin{tabular}{|c|c|c|c|c|c|}
\hline$\alpha=0$ & & $\frac{1}{-2}$ & $\left.n^{-\frac{1}{2}}\right)$ & $2 \sqrt{\log n}$ & $\infty$ \\
\hline new & 1 (Thm. 2) & $\Theta(1)$ (Cor. 2) & $2^{O(\sqrt{\log n})}($ Thm. 4) & $<4$ (Cor. 4) & $\leq 2$ \\
\hline old & \multicolumn{3}{|c|}{$O\left(n^{2 / \alpha}\right)$} & $O\left(\min \left\{4 \sqrt{\log n},(n / \alpha)^{1 / 3}\right\}\right)$ & $\leq 2$ \\
\hline
\end{tabular}

Table 2 Summary of the best known bounds for the price of anarchy in SUMGAME

\begin{tabular}{|c|c|c|c|c|c|c|c|c|}
\hline$=0$ & & & 3 & $\overline{\imath / 2}$ & $\sqrt{n / 2}$ & $\left.n^{1-\epsilon}\right)$ & $12 n$ & $\lg n$ \\
\hline & & $\leq \frac{4}{3}[8]$ & $\leq 4[6]$ & $\leq 6[6]$ & $\Theta(1)[6]$ & $2^{O(\sqrt{\log n})}[6$ & $<5$ (Thm. 7) & $\leq 1.5[1$ \\
\hline
\end{tabular}

behaviour of players, in which players alternately change their strategies to best/better responses [12, 13].

There are other variants of the network creation game, which has little or no impact on the study of the original network creation games, see e.g., [3-5, 7].

\subsection{Our Results}

We first show that computing a best response of a player in MAXGAME is an NPhard problem. In subsequent sections we study price of anarchy of MAXGAME and SumGAME. For MAXGAME we show that price of anarchy is constant for $\alpha>129$ and $\alpha=O\left(n^{-1 / 2}\right)$, and also prove that price of anarchy is $2^{O(\sqrt{\log n})}$ for any $\alpha>0$ in Sect. 3. The result for $\alpha>129$ is obtained as a corollary of the more general result (proven in Sect. 3.1) showing that in MAXGAME for $\alpha>129$ all equilibrium graphs are trees. This is proven by developing new techniques which establish lower and upper bounds on the average degree of biconnected components of equilibrium graphs. In Sect. 4 we adopt the new techniques developed for MAXGAME to prove that for $\alpha>273 n$ all equilibrium graphs of SUMGAME are trees. This result implies a constant upper bound on price of anarchy for $\alpha>273 n$ which shrinks the range of edge-prices for which we do not know a constant upper bound on price of anarchy to $\alpha \in \Omega\left(n^{1-\epsilon}\right) \cap O(n)$. A comparison and overview of the previously known bounds and the new bounds on price of anarchy in both game variants are summarized in Tables 1 and 2.

\section{Complexity of Computing Best Response in MAXGAME}

Computing a best response in SUMGAME was shown to be NP-hard by Fabrikant et al. in [8]. We adapt their proof to show that this is also the case for MAXGAME. One of the consequences is that, unless $P=N P$, computing a Nash equilibrium with a best-response dynamic is not a tractable approach. Moreover, players actually cannot even say, given what every player plays, if they are in a Nash equilibrium.

Theorem 1 Given a player $i \in N$ and a strategy-profile $s \in S$ of MAXGAME, it is $N P$-hard to compute a best response of player $i$. 
Proof Given an undirected graph $G$, DominatingSet is a problem to find a set $D \subseteq V$ of minimum cardinality such that every vertex $v \in V$ has a neighbor in $D$ or is in $D$ itself. This is a well-known NP-hard problem [9]. It is not difficult to see that the problem remains NP-hard for instances where $|D|<n / 2$. Let us denote this problem as DominatingSeT-HALF. We reduce this NP-hard problem to the problem of computing a best response of player $i$ in MAXGAME.

We consider the following instance for the problem of computing a best response in the following MAXGAME: There are $n+1$ players, we set $\alpha=2 / n$ and we set strategies $s_{j}$ of players $j \neq n+1$ such that the graph induced by the strategies of the first $n$ players is $G$, the input graph for DominatingSET-HALF; We ask for a best response of player $n+1$. For any best response $s_{n+1}^{*}$ of player $n+1$ we define $s^{*}=\left(s_{1}, \ldots, s_{n}, s_{n+1}^{*}\right)$. It follows that $D_{G\left(s^{*}\right)}(n+1)<3$ in the resulting graph (recall that $\alpha=2 / n$ and thus buying edges to all other nodes would be a strictly better strategy of player $n+1$ ). Hence, player $n+1$ either buys (i) an edge to every vertex in $N \backslash\{n+1\}$ or (ii) an edge to every vertex of a minimum dominating set in $G$. But since the size of a minimum dominating set in $G$ is less than $n / 2$ and $\alpha=2 / n$, (i) never occurs as a best response.

Therefore finding a best response for player $n+1$ is equivalent to finding a minimum dominating set $D$ in $G$.

\section{Bounding the Price of Anarchy in MaxGame}

In this section we consider MAXGAME. First we classify social optima. This is rather a folklore and resembles in many aspects the previously shown characterization of social optima in SUMGAME. We use this to bound price of anarchy in MAXGAME for small values of $\alpha$.

Proposition 1 For $\alpha \leq \frac{2}{n-2}$ the complete graph is a social optimum. For $\alpha \geq \frac{2}{n-2}$ the star is a social optimum.

Proof Observe that every social optimum $G$ contains a star as a spanning tree (if not, then $D(v) \geq 2$ for every vertex $v$ in $G$ and thus the social cost of $G$ is at least $\alpha|E|+2 n$, which is greater than $\alpha(n-1)+2 n-1$, the social cost of a star). In a star (with $n-1$ edges), one vertex has usage cost 1 . To shorten the usage cost (from 2 to 1 ) of additional vertex $v$ in a star we have to add $n-2$ edges (adjacent to $v$ ) to the star. In general, to have $k$ vertices of usage cost 1 , the graph $G$ has to contain at least $\sum_{i=1}^{k}(n-i)$ edges. Thus, for a fixed value of $\alpha$, the cost of a social optimum is the minimum value of $S C(\alpha, k, n):=\alpha \sum_{i=1}^{k}(n-i)+k+2(n-k)$ among all $1 \leq k \leq n$. Since $\frac{\partial^{2}}{\partial k^{2}} S C(\alpha, k, n)=-\alpha<0$ it either holds $k=1$ or $k=n$ in the minimum. Hence the social optimum is either a complete graph or a star for all values of $\alpha$ and we have $C(\operatorname{Star}) \geq C\left(K_{n}\right) \Leftrightarrow \alpha(n-1)+2 n-1 \geq \alpha n(n-1) / 2+n \Leftrightarrow 2 /(n-2) \geq \alpha$ which proves the claim.

Theorem 2 For $\alpha<\frac{1}{n-2}$ the price of anarchy is 1 . For $\alpha<\frac{2}{n-2}$ the price of anarchy is at most 2. 
Proof By Proposition 1, for $\alpha<\frac{2}{n-2}$, the social optimum is a complete graph. Hence, price of anarchy is at most

$$
\frac{\alpha n(n-1) / 2+n \cdot \operatorname{diam}(G)}{\alpha n(n-1) / 2+n} \leq \operatorname{diam}(G)
$$

for a worst equilibrium graph $G$. First we show that for $\alpha<\frac{1}{n-2}$ every equilibrium graph has diameter 1, i.e. it is a complete graph. Assume for contradiction there is an equilibrium graph $G=(V, E)$ with a vertex $v \in V$ with $D_{G}(v) \geq 2$. Since the graph is connected there are at most $n-2$ vertices with distance more than 1 from $v$. By buying edges to these vertices $v$ can decrease its usage cost by at least 1 . The price for this is $\alpha(n-2)<1$. Thus, $v$ can improve its cost by doing so, a contradiction that $G$ is Nash equlibrium.

Next we show that for $\alpha<\frac{2}{n-2}$ every equilibrium graph has diameter at most 2 . Assume for contradiction there is an equilibrium graph $G=(V, E)$ with a vertex $v \in V$ with $D_{G}(v) \geq 3$. Since the graph is connected there are at most $n-2$ vertices with distance more than 1 from $v$. By buying edges to these vertices $v$ can decrease its usage cost by at least 2. Since $\alpha(n-2)<2, v$ could improve its cost by doing so, a contradiction with the assumption that $G$ is Nash equlibrium.

Next we relate the diameter of an equilibrium graph with price of anarchy of the game, where the following lemma is the key ingredient. The lemma exploits that a breadth-first search tree of an equilibrium graph already contains much information about the whole graph. For SUMGAME a similar result with a similar proof is known [1].

Lemma 1 If $G=(V, E)$ is an equilibrium graph then $C(G) \leq(2 \alpha+1)(n-1)+n$. $\operatorname{rad}(G)$.

Proof Let $T$ be a breadth-first search tree of $G$ rooted in a central vertex $v_{0}$ of $G$. Let $v \in V \backslash\left\{v_{0}\right\}$. Let $E_{v}$ be the edges built by $v$ in $T$. Consider the following strategy of $v$ : Buy all edges of $E_{v}$ plus buy the edge to $v_{0}$. The creation cost of $v$ in this strategy is at most $\alpha\left(\left|E_{v}\right|+1\right)$ and the usage cost is at most $D\left(v_{0}\right)+1$. As $G$ is an equilibrium, every vertex (player) achieves in $G$ the best possible cost, given what other players do. Thus, the above mentioned strategy upper-bounds the cost of $v$ in equilibrium, i.e., $c_{v}(G) \leq \alpha\left(\left|E_{v}\right|+1\right)+\operatorname{rad}(G)+1$. For vertex $v_{0}$ we have $c_{v_{0}}(G)=$ $\alpha\left|E_{v_{0}}\right|+\operatorname{rad}(G)$. Summing the obtained inequalities for every vertex of $G$ yields the claimed inequality.

Corollary 1 Let $G$ be a worst Nash equilibrium for $\alpha \geq \frac{2}{n-2}$. The price of anarchy is $O\left(1+\frac{\operatorname{diam}(G)}{\alpha+1}\right)$.

Proof By Proposition 1 and Lemma 1 we get that price of anarchy is at most

$$
\frac{(2 \alpha+1)(n-1)+n \cdot \operatorname{rad}(G)}{(\alpha+2)(n-1)+1} \leq \frac{2 \alpha+1}{\alpha+2}+\frac{n \cdot \operatorname{rad}(G)}{(n-1)(\alpha+2)} \leq 2+\frac{2 \cdot \operatorname{rad}(G)}{\alpha+2} .
$$


Demaine et al. [6] showed that the diameter of equilibrium graphs is bounded by $O\left(1+\alpha 4^{\sqrt{\lg n}}\right)$ and by $O\left(1+\left(n \alpha^{2}\right)^{1 / 3}\right) \cdot{ }^{3}$ Combining these results with Corollary 1 yields an improved bound for the price of anarchy:

Lemma 2 [6] The diameter of an equilibrium graph is $O\left(1+\left(n \alpha^{2}\right)^{1 / 3}\right)$.

Theorem 3 For $\alpha=O(1)$ the price of anarchy is $O\left(1+\left(n \alpha^{2}\right)^{1 / 3}\right)$.

Proof This is an immediate consequence of Lemma 3 and Corollary 1.

Corollary 2 For $\alpha=O\left(n^{-1 / 2}\right)$ the price of anarchy is constant.

Lemma 3 [6] The diameter of an equilibrium graph is $O(1+\alpha \cdot 4 \sqrt{\lg (n)})$.

Theorem 4 The price of anarchy is $2^{O(\sqrt{\log (n)})}$.

Proof This is an immediate consequence of Lemma 3 and Corollary 1.

In the following we show that equilibrium graphs that are trees have cost at most a constant times bigger than the cost of a social optimum. Thus, if for given $\alpha$ all equilibrium graphs are trees, then price of anarchy is constant. We note that a similar result for SuMGaME has been shown by Fabrikant et al. [8]. We show in Sect. 3.1 that for $\alpha>129$ all equilibrium graphs are trees which shows that price of anarchy for this range of $\alpha$ is constant.

Theorem 5 The cost of an equilibrium graph that is a tree is less than 4 times the cost of a social optimum.

Proof Observe that the claim is trivial when $n \leq 2$, or when $\alpha<2 /(n-2)$ (as then, by Theorem 2, price of anarchy is at most 2). We therefore assume that $n \geq 3$ and $\alpha \geq$ $2 /(n-2)$. Let $T=(V, E)$ be a tree on $n \geq 3$ vertices that is Nash equilibrium. We first show that $\operatorname{diam}(T) \leq 2 \alpha+3$. Let $v \in V$ be a vertex with $D(v)=\lceil\operatorname{diam}(T) / 2\rceil$ (observe that there exists such a vertex). Consider $T$ rooted at $v$. Let $l$ be a leaf of $T$ at depth $D(v)$. Consider the strategy of $l$ where $l$ buys, additionally to what it does in the equilibrium strategy profile, an edge to $v$. The usage cost of $l$ is at most $1+D(v)$ using the new strategy. Its usage cost in the equilibrium strategy is $D(l)=\operatorname{diam}(T)$. As $T$ is Nash equilibrium we can conclude that buying the edge to $v$ is not beneficial and therefore $\alpha \geq D(l)-(D(v)+1) \geq\lfloor\operatorname{diam}(T) / 2\rfloor-1$. Hence, $\operatorname{diam}(T) \leq 2 \alpha+3$. We now compare the cost of $T$ with the cost of a social optimum $G_{\mathrm{OPT}}$. As $\alpha \geq 2 /(n-2)$, a star is a social optimum (Proposition 1$)$. Hence, as $C\left(G_{\mathrm{OPT}}\right)=$

\footnotetext{
${ }^{3}$ In fact, [6] claims a bound of $O\left(\alpha 4^{\sqrt{\lg n}}\right)$ resp. $O\left(\left(n \alpha^{2}\right)^{1 / 3}\right)$ on the diameter, which does not make sense for very small $\alpha$. The arguments given in [6] show a bound of $O\left(1+\alpha 4^{\sqrt{\lg n}}\right)$ resp. $O\left(1+\left(n \alpha^{2}\right)^{1 / 3}\right)$ on the diameter.
} 


$$
\begin{aligned}
& (\alpha+2)(n-1)+1, \\
& \frac{C(T)}{C\left(G_{\mathrm{OPT}}\right)} \leq \frac{\alpha(n-1)+\operatorname{diam}(T) \cdot n}{(\alpha+2)(n-1)} \leq \frac{\alpha}{\alpha+2}+\frac{(2 \alpha+3) \cdot n}{(\alpha+2)(n-1)}<1+2 \cdot \frac{3}{2}=4,
\end{aligned}
$$

which proves the claim.

\subsection{For $\alpha>129$ Every Equilibrium Graph is a Tree}

In this section we present the main result for MAXGAME, namely, we show that for $\alpha>129$ every equilibrium graph is a tree. This, together with Theorem 5 , shows that price of anarchy is smaller than 4 for this range of $\alpha$. The main idea is to show that an arbitrary (non trivial) biconnected component of an equilibrium graph has average degree $c>2$ and at the same time smaller than $2+\frac{c^{\prime}}{\alpha}$ for some constants $c, c^{\prime}$. For big enough $\alpha$ these inequalities become contradicting and thus we know that this cannot happen, i.e., every Nash equilibrium for such $\alpha$ contains no biconnected component other than bridges and therefore no cycle-it has to be a tree.

For the entire section let $G=(V, E)$ be a graph on $n$ vertices that contains at least one cycle and let $H \subseteq G$ be an (arbitrary) biconnected component of $G$ of size $|H| \geq 3$. Furthermore we use the following definitions. For a vertex $v \in V$ and a set $X \subseteq V$ we call a path starting in $v$ and ending in a vertex in $X$ a $v$ - $X$-path. For every vertex $v$ in $H$ we define $S(v)$ to be the set of all vertices $x \in V$ such that a shortest $x$ - $H$-path ends in $v$. Note that by definition: $S(v) \neq \emptyset$ since $v \in S(v) ; v$ is the only vertex from $H$ in $S(v) ; S(u) \cap S(v)=\emptyset$ for $u \in V(H), u \neq v$; for every $w \in S(v)$ every shortest $u$ - $w$-path contains $v$.

We start with the observation of Demaine et al. [6] that the edge price bounds the girth of equilibrium graphs from below:

Lemma 4 [6] Every equilibrium graph has no cycle of length less than $\alpha+2$.

Proof Suppose for contradiction that there is a cycle $C$ with $|C|<\alpha+2$. Let $\{u, v\}$ be an edge in $C$ and without loss of generality assume that $u$ bought it. If $u$ removes this edge it increases its usage cost by at most $|C|-2<\alpha$. Hence $u$ should not buy the edge and we have a contradiction.

The following lemma shows that the usage cost of vertices in $H$ differ by at most 4 and "tends to be lower" for a vertex that buys an edge in $H$.

Lemma 5 If $G$ is an equilibrium graph and $v \in V(H)$ then $D_{G}(v) \leq \operatorname{rad}(G)+3$ if $v$ buys an edge in $H$ and $D_{G}(v) \leq \operatorname{rad}(G)+4$ otherwise.

Proof We show that for every edge $\{u, v\} \in E(H)$ bought by $u$ we have $D_{G}(u) \leq$ $\operatorname{rad}(G)+3$ and $D_{G}(v) \leq \operatorname{rad}(G)+4$. The claim then follows. Consider a breadthfirst search tree $T$ rooted in some central vertex $v_{0}$ of $G$. First we consider the case that $\{u, v\} \in E(H) \backslash E(T)$. Trivially, $D_{G}(u) \leq \operatorname{rad}(G)+1$ (as otherwise $u$ could buy an edge $\left\{u, v_{0}\right\}$ instead of $\{u, v\}$ and thus improve its cost) and therefore $D_{G}(v) \leq$ $\operatorname{rad}(G)+2$. Next we consider the case that $\{u, v\} \in E(T) \cap E(H)$. We note that the 
edge either leads "up" the tree to $v_{0}$, or it leads "down" the tree such that there is a vertex $s \in V(H)$ below or at $v$ which is incident to an edge in $E(H) \backslash E(T)$ (if not then $u$ would be a cut vertex of $H$ ). In the first case we have $D_{G}(u) \leq \operatorname{rad}(G)+1$ (as otherwise $u$ could buy an edge $\left\{u, v_{0}\right\}$ instead of $\{u, v\}$ and thus improve its cost). In the second case we have, as shown before, $D_{G}(s) \leq \operatorname{rad}(G)+2$ and therefore $D_{G}(u) \leq \operatorname{rad}(G)+3$ (as otherwise $u$ could buy an edge $\{u, s\}$ instead of $\{u, v\}$ and thus improve its cost). So in general we have $D_{G}(u) \leq \operatorname{rad}(G)+3$ and therefore $D_{G}(v) \leq \operatorname{rad}(G)+4$.

In the following lemmas we show that for every vertex in a biconnected component $H$ of an equilibrium graph $G$ there is a vertex of degree at least 3 in $H$ in a constantsize neighborhood of $v$.

Lemma 6 If $G$ is an equilibrium graph for $\alpha>0$ then for every vertex $v$ in $H$ and every vertex $w \in S(v): d_{G}(v, w) \leq \operatorname{rad}(G)+\frac{7-\alpha}{2}$.

Proof By Lemma 4, $H$ has no cycle of length less than $\alpha+2$. Thus, as every vertex of $H$ is contained in at least one cycle, there is a vertex $u \in V(H)$ with $d_{G}(u, v)=$ $d_{H}(u, v) \geq\left\lfloor\frac{\alpha+2}{2}\right\rfloor \geq \frac{\alpha+1}{2}$. Every shortest $u$-w-path contains vertex $v$ (by definition of $S(v))$. Therefore $d_{G}(u, w)=d_{G}(u, v)+d_{G}(v, w) \geq \frac{\alpha+1}{2}+d_{G}(v, w)$. By Lemma 5 we have $d_{G}(u, w) \leq D_{G}(u) \leq \operatorname{rad}(G)+4$. Hence $d_{G}(v, w) \leq \operatorname{rad}(G)+\frac{7-\alpha}{2}$.

Lemma 7 If $G$ is an equilibrium graph for $\alpha>11$, then for every vertex $v$ in $H$ that buys at least two edges in $H$ there is a vertex $w \in N_{1}(v)$ with $\operatorname{deg}_{H}(w) \geq 3$.

Proof Let us refer to $v$ by $x_{2}$ and let $x_{1}$ and $x_{3}$ be two vertices to which $x_{2}$ buys edges in $H$. Assume for contradiction that $\operatorname{deg}_{H}\left(x_{i}\right)=2$ for $i=1,2,3$. Denote the $x_{1}$ 's other neighbor in $H$ by $x_{0}$ and the $x_{3}$ 's other neighbor in $H$ by $x_{4}$. Note that, as $\alpha>11$, the girth of $H$ is at least 14 (Lemma 4) and therefore $x_{i} \neq x_{j}$ for $i \neq j$. Also by Lemma 6 we have $d_{G}\left(x_{2}, w\right)<\operatorname{rad}(G)-1 \leq D_{G}\left(x_{2}\right)-1$ for $w \in \bigcup_{i=1,2,3} S\left(x_{i}\right)$. Thus, all shortest $x_{2}-\mathcal{E}\left(x_{2}\right)$-paths contain either $x_{0}$ or $x_{4}$. Hence, by buying edges to $x_{0}$ and $x_{4}$ instead of $x_{1}$ and $x_{3}, x_{2}$ would decrease its distance to the vertices in $\mathcal{E}\left(x_{2}\right)$, increase its distance to the vertices in $S\left(x_{1}\right)$ and $S\left(x_{3}\right)$ by at most 1 and it would not increase its distance to any other vertex. Therefore (as $d_{G}\left(x_{2}, w\right)<D_{G}\left(x_{2}\right)-1$ for $\left.w \in S\left(x_{1}\right) \cup S\left(x_{3}\right)\right)$, by changing its strategy $x_{2}$ could improve. But this contradicts equilibrium and hence we have $\operatorname{deg}_{H}\left(x_{i}\right) \geq 3$ for some $i \in\{1,2,3\}$.

Lemma 8 If $G$ is an equilibrium graph for $\alpha>13$ then any path $x_{0}, x_{1}, \ldots, x_{k}$ in $H$ with $\operatorname{deg}_{H}\left(x_{i}\right)=2$ for $0 \leq i \leq k$ such that for $0 \leq i<k,\left\{x_{i}, x_{i+1}\right\}$ is bought by $x_{i}$, has length at most $k \leq 4$.

Proof Consider a maximal path $x_{0}, x_{1}, \ldots, x_{k}$ in $H$ of the form from the statement and assume for contradiction $k \geq 5$. By Lemma 5 we have $\left|D_{G}\left(x_{i}\right)-D_{G}\left(x_{j}\right)\right| \leq 3$ for $0 \leq i, j \leq k-1$ and therefore, by the pigeonhole principle, there is $0 \leq i_{0} \leq 3$ such that $D_{G}\left(x_{i_{0}}\right) \geq D_{G}\left(x_{i_{0}+1}\right)$. Denote the $x_{i_{0}+2}$ 's other neighbor in $H$ by $x_{i_{0}+3}$ (if not already so denoted). For every vertex $w \in S\left(x_{i_{0}+j}\right), j=0,1,2$, we have (using 
Lemma 6) $d_{G}\left(x_{i_{0}+j}, w\right)<\operatorname{rad}(G)-3$, and therefore $\mathcal{E}\left(x_{i_{0}}\right) \cap S\left(x_{i_{0}+j}\right)=\emptyset$ for $j=$ $0,1,2$.

We consider the strategy where $x_{i_{0}}$ buys an edge to $x_{i_{0}+3}$ instead of the edge to $x_{i_{0}+1}$ and show that $x_{i_{0}}$ improves in this strategy, which is a contradiction. We split the vertices of $\mathcal{E}\left(x_{i_{0}}\right)$ into two parts: set $S$ where for every $z \in S$ no shortest $x_{i_{0}}$-z-path contains $x_{i_{0}+1}$, and set $\mathcal{E}\left(x_{i_{0}}\right) \backslash S$ where for every vertex $z \in \mathcal{E}\left(x_{i_{0}}\right) \backslash S$ there is a shortest $x_{i_{0}}$-z-path that contains $x_{i_{0}+1}$ (and therefore also $x_{i_{0}+2}$ and $x_{i_{0}+3}$ ). Observe that in the new strategy $x_{i_{0}}$ decreases its distance to vertices in $\mathcal{E}\left(x_{i_{0}}\right) \backslash S$ by 2 , and increases its distance to vertices in $S\left(x_{i_{0}+1}\right)$ by at most 2 , and does not increase its distance to any other vertex of $V$ but perhaps to those in $S$. We show that $x_{i_{0}}$ actually decreases its distance to every vertex in $S$ by at least one, which shows that $x_{i_{0}}$ improves in the new strategy (recall that $d_{G}\left(x_{i_{0}}, y\right)<D_{G}\left(x_{i_{0}}\right)-2$ for every $y \in S\left(x_{i_{0}+1}\right)$ ). To show that $x_{i_{0}}$ improves its distance to every vertex $z \in S$, we first observe that because $D_{G}\left(x_{i_{0}}\right) \geq D_{G}\left(x_{i_{0}+1}\right)$ no shortest $x_{i_{0}+1}-z$-path contains $x_{i_{0}}$. Thus, all shortest

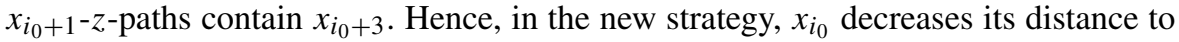
$z$, which finishes the proof.

Lemma 9 If $G$ is an equilibrium graph for $\alpha>13$ then for every vertex $v$ in $H$ there is a vertex $w \in N_{5}(v)$ with $\operatorname{deg}_{H}(w) \geq 3$.

Proof Let $\{u, v\}$ be an arbitrary edge in $H$ and assume without loss of generality that $u$ bought the edge. Let $C$ be a cycle containing $\{u, v\}$ and note that by Lemma 4 it has at least 16 vertices. Denote the vertices after $v$ and $u$ (in that order) in $C$ by $x_{0}, x_{1}, x_{2}, \ldots$ We distinguish two cases. Assume first that there is a vertex $y \in\left\{u, x_{0}, x_{1}, x_{2}\right\}$ that buys both its edges in $C$. Then, by Lemma 7 , there is vertex $w \in N_{1}(y) \subseteq N_{4}(u) \subseteq N_{5}(v)$ with $\operatorname{deg}_{H}(w) \geq 3$. Assume now that there is no vertex $y \in\left\{u, x_{0}, x_{1}, x_{2}\right\}$ that buys both its edges in $C$. But then, as $u$ buys an edge to $v$, we have a path $x_{3}, x_{2}, x_{1}, x_{0}, u, v$ of length 5 where one vertex buys the edge to the next one. Thus, by Lemma 8 , the vertices of the path cannot have all degree 2 in $H$, and the lemma follows.

Corollary 3 If $G$ is an equilibrium graph for $\alpha>13$ then $\operatorname{deg}(H) \geq 2+\frac{1}{16}$.

Proof We assign every vertex $v \in H$ to its closest vertex $c \in H$ with $\operatorname{deg}_{H}(c) \geq 3$ (thus, $c$ is assigned to itself), breaking ties arbitrarily (by Lemma 9 we know that there is a vertex of degree at least 3 in $H$ ). Consider the subgraph of $H$ formed by a vertex $c$ of degree at least 3 and by vertices assigned to it. Observe that these subgraphs form a partition of $H$. We show that the average degree of every such subgraph is at least $2+\frac{1}{16}$ which proves the claim. The subgraph consists of $\operatorname{deg}_{H}(c)$ induced paths $\left\{p_{i}(c)\right\}_{i=1}^{\operatorname{deg}_{H}(c)}$ that all meet in $c$. Let length $\left(p_{i}(c)\right)$ denote the length of path $p_{i}(c)$. By Lemma 9 this length is at most 5 . The average degree of the subgraph is then $\frac{\operatorname{deg}_{H}(c)+2 \sum_{i=1}^{\operatorname{deg}_{H}(c)} \text { length }\left(p_{i}(c)\right)}{1+\sum_{i=1}^{\operatorname{deg}_{H}(c)} \text { length }\left(p_{i}(c)\right)}=2+\frac{\operatorname{deg}_{H}(c)-2}{1+\sum_{i=1}^{\operatorname{deg}_{H}(c)} \text { length }\left(p_{i}(c)\right)} \geq 2+\frac{\operatorname{deg}_{H}(c)-2}{1+5 \cdot \operatorname{deg}_{H}(c)} \geq$ $2+\frac{1}{16}$.

Next we prove the last ingredient for our approach-we show an upper bound for $\operatorname{deg}(H)$ involving $\alpha$ : 
Lemma 10 If $G$ is an equilibrium graph for $\alpha>1$ then $\operatorname{deg}(H) \leq 2+\frac{8}{\alpha-1}$.

Proof Consider a breadth-first search tree $T$ of $G$ rooted in a central vertex $v_{0} \in$ $V$ and let $\tilde{T}:=T \cap H$. Note that $\tilde{T}$ is a spanning tree of $H$. Then $\operatorname{deg}(H)=$ $\frac{2|E(\tilde{T})|+2|E(H) \backslash E(\tilde{T})|}{|V(\tilde{T})|} \leq 2+\frac{2|E(H) \backslash E(\tilde{T})|}{|V(\tilde{T})|}$, and hence we have to bound $|E(H) \backslash E(\tilde{T})|$ (the number of edges outside $\tilde{T}$ ). To do that, we consider vertices of $H$ that buy an edge in $E(H) \backslash E(\tilde{T})$. Let us call such a vertex a shopping vertex. First observe that every shopping vertex $u$ buys exactly one edge in $E(H) \backslash E(\tilde{T})$, as otherwise $u$ could opt not to buy these edges and buy one edge to $v_{0}$ instead, thus saving at least $\alpha$ on creation cost, and having usage cost at most $D_{G}\left(v_{0}\right)+1 \leq D_{G}(u)+1$, which (for $\alpha>1$ ) would be an improvement, a contradiction. This immediately shows that there are at most $|V(\tilde{T})|$ edges in $E(H) \backslash E(\tilde{T})$. To get a better bound, we bound the number of shopping vertices. We show that the distance in $\tilde{T}$ between any two shopping vertices is at least $\frac{\alpha-1}{2}$. The upper bound on the number of shopping vertices follows: Assign every node $v$ of $H$ to the closest shopping vertex (closest according to the distance in $\tilde{T}$; breaking ties arbitrarily); Observe that this assignment forms a partition of $H$ (and that every part contains exactly one shopping vertex); As the distance in $\tilde{T}$ between any two shopping vertices is at least $\frac{\alpha-1}{2}$, the size of every part is at least $\frac{\alpha-1}{4}$. Thus, there are at most $\frac{4|V(\tilde{T})|}{\alpha-1}$ shopping vertices and thus at most that many edges in $E(H) \backslash E(\tilde{T})$; The desired bound $\operatorname{deg}(H) \leq 2+\frac{8}{\alpha-1}$ now easily follows.

We are left to prove that the distance in $\tilde{T}$ between any two shopping vertices is at least $\frac{\alpha-1}{2}$. Assume for contradiction that there are two shopping vertices $u_{1} \neq u_{2}$ for which $d_{\tilde{T}}\left(u_{1}, u_{2}\right)<\frac{\alpha-1}{2}$. Let $u_{1}=x_{1}, x_{2}, \ldots, x_{k}=u_{2}$ be the shortest $u_{1}-u_{2}$ path in $\tilde{T}$ and let us call it $P$. Let $\left\{u_{1}, v_{1}\right\}$ and $\left\{u_{2}, v_{2}\right\}$ be the edges that $u_{1}$ and $u_{2}$ buy in $E(H) \backslash E(\tilde{T})$. Observe that $v_{1}$ and $v_{2}$ are not descendant of any vertex $x_{i}$, $i=1, \ldots, k$, in $P$; If $v_{j}, j=1,2$, is descendant of $x_{i}$, then the $v_{j}-x_{i}$-path in $\tilde{T}$, the $x_{i}$ $u_{j}$-path in $\tilde{T}$, and the edge $\left\{u_{j}, v_{j}\right\}$ form a cycle of length at most $2\left(d_{\tilde{T}}\left(u_{1}, u_{2}\right)+1\right)<$ $\alpha+1$ which contradicts Lemma 4. In particular, $v_{j}$ is not part of $P$, and therefore $x_{0}=$ $v_{1}, x_{1}, \ldots, x_{k}, x_{k+1}=v_{2}$ is a path in $H$. Also by Lemma $4, u_{j}, j=1,2$, has distance at least $\frac{\alpha-1}{2}$ from $v_{0}$, and therefore $v_{0}$ is not in $P$. Now, since $x_{1}$ buys $\left\{x_{0}, x_{1}\right\}$ and $x_{k}$ buys $\left\{x_{k}, x_{k+1}\right\}$, there has to be $1 \leq i^{*} \leq k$ such that $x_{i *}$ buys both $\left\{x_{i^{*}-1}, x_{i *}\right\}$ and $\left\{x_{i^{*}}, x_{i^{*}+1}\right\}$. Consider the following modification of $x_{i^{*}}$ 's strategy: Buy edge $\left\{x_{i^{*}}, v_{0}\right\}$ instead of edges $\left\{x_{i^{*}-1}, x_{i^{*}}\right\}$ and $\left\{x_{i^{*}}, x_{i^{*}+1}\right\}$. In this new strategy, $x_{i^{*}}$ decreases its creation cost by $\alpha$. We now show that $x_{i *}$ 's new usage cost is $D_{\text {new }}\left(x_{i *}\right)<D_{G}\left(x_{i^{*}}\right)+$ $\alpha$ thus implying that the new strategy improves $x_{i *}$ 's cost, a contradiction.

First note that $D_{\text {new }}\left(x_{i}{ }^{*}\right) \leq 1+D_{\text {new }}\left(v_{0}\right)$ (where the subscript "new" always corresponds to the situation in a graph where $x_{i}$ is using the modified strategy). To bound $D_{\text {new }}\left(v_{0}\right)$ we note that only the vertices in $P$ and their descendants in $T$ can have increased distance to $v_{0}$ by the strategy change. Let $y$ be one of these vertices with possibly increased distance and let $1 \leq j \leq k$ be such that $x_{j}$ is the closest ancestor of $y$, i.e., an ancestor with $d_{G}\left(x_{j}, y\right)=\min _{x \in P} d_{G}(x, y)$. If $j=i^{*}$ it is easy to see that $d_{\text {new }}\left(v_{0}, y\right) \leq d_{G}\left(v_{0}, y\right)$ and therefore for such a vertex $y$ there is no increase in usage cost of $v_{0}$. Consider now the case $j \neq i^{*}$ and assume (without loss of generality, as we shall see) that $j<i^{*}$. Then $d_{\text {new }}\left(v_{0}, y\right) \leq d_{\text {new }}\left(v_{0}, x_{0}\right)+d_{\text {new }}\left(x_{0}, x_{j}\right)+d_{\text {new }}\left(x_{j}, y\right)=$ 
$d_{G}\left(v_{0}, x_{0}\right)+d_{G}\left(x_{0}, x_{j}\right)+d_{G}\left(x_{j}, y\right)$ (since $x_{0}$ is not a descendant of a vertex in $P$ and $x_{0}, \ldots, x_{j}$ is still a path in $\left.G_{\text {new }}\right)$, and $d_{G}\left(v_{0}, y\right)=d_{G}\left(v_{0}, x_{j}\right)+d_{G}\left(x_{j}, y\right)$. Then the increase of usage cost of $v_{0}$ is: $d_{\text {new }}\left(v_{0}, y\right)-d_{G}\left(v_{0}, y\right)=d_{G}\left(v_{0}, x_{0}\right)+d_{G}\left(x_{0}, x_{j}\right)-$ $d_{G}\left(v_{0}, x_{j}\right) \leq 2 \cdot d_{G}\left(x_{0}, x_{j}\right) \leq 2 \cdot d_{G}\left(u_{1}, u_{2}\right) \leq 2 \cdot d_{\tilde{T}}\left(u_{1}, u_{2}\right)<\alpha-1$, where the last inequality follows from our assumption $d_{\tilde{T}}\left(u_{1}, u_{2}\right)<\frac{\alpha-1}{2}$. As $y$ was chosen arbitrary, we have that the increase of usage cost of $v_{0}$ is less than $\alpha-1$ and therefore $D_{\text {new }}\left(v_{0}\right)<D_{G}\left(v_{0}\right)+\alpha-1$, which shows $D_{\text {new }}\left(x_{i^{*}}\right)<D_{G}\left(x_{i *}\right)+\alpha$.

We are now ready to state the threshold on $\alpha$, above which only tree equilibria appear.

Theorem 6 For $\alpha>129$ every equilibrium graph is a tree.

Proof If $G$ is a non-tree equilibrium for $\alpha>129$ and $H$ a block in $G$ with $|H| \geq 3$ then we have by Lemma 10 that $\operatorname{deg}(H) \leq 2+\frac{8}{\alpha-1}<2+\frac{1}{16}$, which contradicts Corollary 3 stating that $\operatorname{deg}(H) \geq 2+\frac{1}{16}$.

This bound is asymptotically tight. Indeed there is a constant $c>0$ such that for $\alpha<c$ we have non-tree equilibrium graphs. E.g., for $\alpha \leq 1$, the triangle is an equilibrium graph (we can generalize this to any size $n \geq 3$ of vertices: three stars of size $n / 3$, where the three centers of the stars are connected in a triangle, form an equilibrium graph, too). Theorems 6 and 5 thus show the following.

Corollary 4 For $\alpha>129$ the price of anarchy is smaller than 4.

Proof This is an immediate consequence of Theorems 6 and 5.

\section{Bounding the Price of Anarchy in SumGame}

In this section we consider SumGame. Adapting the methods that we have developed for MAXGAME in Sect. 3.1 we are able to show that in SUMGAME for $\alpha>273 n$ every equilibrium graph is a tree. This improves the best known bound of $\alpha \geq 12 n \lg n$ from [1] and is asymptotically the best obtainable bound as for $\alpha<n / 2$ there exist non-tree equilibrium graphs [1]. As a corollary we obtain constant price of anarchy for $\alpha>273 n$. We use the same conventions and notation as in Sect. 3.1, i.e. $G=(V, E)$ is a graph on $n$ vertices that contains at least one cycle, $H$ is a block of $G$ of size $|H| \geq 3$ and $S(v)$ for $v \in V(H)$ is defined as in Sect. 3.1. The next discussion closely follows the ideas for MAXGAME (Sect. 3.1). Recall that in SuMGAME the usage cost of a vertex $v$ is the sum of all distances from $v$ to any vertex in the resulting graph of the game.

Similarly to MAXGAME we can show in the following lemmas that in a constantsize neighborhood of every vertex $v$ in a biconnected component $H$ of an equilibrium graph $G$ there is a vertex of degree at least 3 in $H$. The details of the proofs are for SUMGAME a bit different though. 
Lemma 11 [1] Any equilibrium graph has no cycle of length less than $\frac{\alpha}{n}+2$.

Proof Suppose for contradiction that there is a cycle $C$ with $|C|<\frac{\alpha}{n}+2$. Let $\{u, v\}$ be an edge in $C$ and without loss of generality assume that $u$ bought it. If $u$ removes this edge it increases its usage cost by at most $n \cdot(|C|-2)<\alpha$. Hence $u$ should not buy the edge and we have a contradiction.

Lemma 12 If $G$ is an equilibrium graph and $u, v \in V(H)$ are two vertices in $H$ with $d(u, v) \geq 3$ such that $u$ buys the edge to its adjacent vertex $x$ in a shortest $u$ - $v$-path and $v$ buys the edge to its adjacent vertex $y$ in that path then either $\operatorname{deg}_{H}(x) \geq 3$ or $\operatorname{deg}_{H}(y) \geq 3$.

Proof Assume for contradiction that $\operatorname{deg}_{H}(x)=2=\operatorname{deg}_{H}(y)$. Assume without loss of generality that $|S(x)| \leq|S(y)|$. Let $z$ be the other vertex in $H$ adjacent to $x$. Consider a modified strategy of $u$ where $u$ buys an edge to $z$ instead of the edge to $x$. In this strategy $u$ shortens its distance to the vertices in $S(y)$ and $S(v)$ by at least 1 and increases its distance to the vertices in $S(x)$ by 1 . Furthermore it does not increase its distance to any other vertex in the graph. Since $|S(x)|<|S(v) \cup S(y)|(S(v) \neq \emptyset$ by definition), we conclude that $u$ decreases its cost in the modified strategy, a contradiction.

The proof of the following lemma is most different from the techniques used for MAXGAME.

Lemma 13 If $G$ is an equilibrium graph then any path $x_{0}, x_{1}, \ldots, x_{k}$ in $H$, where $\operatorname{deg}_{H}\left(x_{i}\right)=2$ for $0 \leq i \leq k$ and $x_{i}$ buys $\left\{x_{i}, x_{i+1}\right\}$ for $0 \leq i \leq k-1$, has length at most $k \leq 8$.

Proof Consider a maximal path $x_{0}, x_{1}, \ldots, x_{k}$ of the form from the statement and assume for contradiction that $k \geq 9$. In the following we denote by $x_{k+1}$ the other neighbor of $x_{k}$ in $H$ (i.e., not $x_{k-1}$ ).

We consider a breadth-first search tree $T$ of $G$ rooted in $x_{k-1}$. We denote by $N$ the set consisting of $x_{k+1}$ and its descendants in $T$. If $x_{k-1}$ would buy $\left\{x_{k-1}, x_{k+1}\right\}$ instead of $\left\{x_{k-1}, x_{k}\right\}$ it would decrease its distance to all vertices in $N$ by at least 1 , increase its distance to all vertices in $S\left(x_{k}\right)$ by at most 1 , and would not increase its distance to any other vertex. Since $G$ is an equilibrium we have $|N| \leq\left|S\left(x_{k}\right)\right|$. Similarly for $1 \leq i \leq k-1$ if $x_{i-1}$ buys $\left\{x_{i-1}, x_{i+1}\right\}$ instead of $\left\{x_{i-1}, x_{i}\right\}, x_{i-1}$ would decrease its distance to all vertices in $\bigcup_{j=i+1}^{\min \{i-1+\lceil k / 2\rceil, k\}} S\left(x_{j}\right)$ by at least 1 , increase its distance to all vertices in $S\left(x_{i}\right)$ by at most 1 , and would not increase its distance to any other vertex. Hence we have

$$
\left|S\left(x_{i}\right)\right| \geq\left|\bigcup_{j=i+1}^{\min \{i-1+\lceil k / 2\rceil, k\}} S\left(x_{j}\right)\right|
$$

and inductively we obtain

$$
\left|S\left(x_{1}\right)\right| \geq\left|S\left(x_{\lfloor k / 2\rfloor+1}\right)\right| \geq 2^{\lceil k / 2\rceil-2}|N| .
$$


Next we show that $\operatorname{deg}_{H}\left(x_{k+1}\right) \geq 3$. By the maximality assumption on the path we must have either $\operatorname{deg}_{H}\left(x_{k+1}\right) \geq 3$ or $x_{k+1}$ buys $\left\{x_{k}, x_{k+1}\right\}$ or $x_{0}=x_{k+1}$. In the second case as $x_{k-2}$ bought an edge to $x_{k-1}$, Lemma 12 implies that $x_{k-1}$ or $x_{k}$ have degree at least 3 in $H$ which is a contradiction. In the third case as $x_{k}$ buys an edge to $x_{0}=x_{k+1}$ we can argue as in the estimations above: If $x_{k}$ would buy $\left\{x_{k}, x_{1}\right\}$ instead of $\left\{x_{k}, x_{0}\right\}$ it would decrease its distance to the vertices in $S\left(x_{1}\right)$ by at least 1 , increase its distance to the vertices in $S\left(x_{0}\right)=S\left(x_{k+1}\right) \subseteq N$ by 1 and would not increase its distance to any other vertex. Hence we have $|N| \geq\left|S\left(x_{0}\right)\right| \geq\left|S\left(x_{1}\right)\right|$. But by (2) we have $\left|S\left(x_{1}\right)\right| \geq 2^{\lceil k / 2\rceil-2}|N|$ a contradiction. So indeed we have $\operatorname{deg}_{H}\left(x_{k+1}\right) \geq 3$.

Now we can consider the following two cases: Either at least one child $u$ of $x_{k+1}$ buys an edge to $x_{k+1}$ or $x_{k+1}$ buys the edges to all its children (of which at least two are in $H$ ). In the first case we propose for $u$ the following change of strategy: It should buy an edge to $x_{k-5}$ instead of $x_{k}$. Thereby $u$ would decrease its distance to the vertices in $S\left(x_{k-3}\right) \cup S\left(x_{k-4}\right) \cup S\left(x_{k-5}\right)$ by at least 2 , it would increase its distance to the vertices in $N \cup S\left(x_{k}\right) \cup S\left(x_{k-1}\right)$ by at most 6 , furthermore since $x_{k-5}$ has depth greater than $u$ it would not increase its distance to any other vertex. Since $G$ is an equilibrium $u$ cannot improve and we have $2\left|S\left(x_{k-3}\right) \cup S\left(x_{k-4}\right) \cup S\left(x_{k-5}\right)\right| \leq 6\left|N \cup S\left(x_{k}\right) \cup S\left(x_{k-1}\right)\right| \leq 18\left|S\left(x_{k-1}\right)\right|$ (where the last inequality follow from $\left.|N| \leq\left|S\left(x_{k}\right)\right| \leq\left|S\left(x_{k-1}\right)\right|\right)$. But this is a contradiction since $\left|S\left(x_{k-3}\right) \cup S\left(x_{k-4}\right) \cup S\left(x_{k-5}\right)\right|>9\left|S\left(x_{k-1}\right)\right|$ by (1).

In the second case we propose for $x_{k+1}$ the following change of strategy: It should buy an edge to $x_{1}$ and delete the edges to its children in $H$. By the change of strategy $x_{k+1}$ would not increase its distance to any vertex in $V \backslash N$ (since $x_{1}$ has depth greater than $x_{k+1}$ as $k \geq 9$ ), it would increase its distance to any vertex in $N$ by at most $2 \cdot \operatorname{diam}(H)$ (deleting the edges does not make the graph disconnected as otherwise $x_{k+1}$ would be a cut vertex of $H$ ) and it would lower its creation cost by at least $\alpha$. Hence since $x_{k+1}$ cannot improve from this change we must have $\alpha \leq$ $2 \operatorname{diam}(H) \cdot|N|$. On the other hand we have $(\operatorname{rad}(H)-1)\left|S\left(x_{1}\right)\right| \leq \alpha$ since otherwise a vertex with distance $\operatorname{rad}(H)$ from $x_{1}$ could improve by buying an edge to $x_{1}$ thereby lowering its usage cost by at least $(\operatorname{rad}(H)-1)\left|S\left(x_{1}\right)\right|$. Combining this inequalities with (2) we obtain: $2^{\lceil k / 2\rceil-2}(\operatorname{rad}(H)-1)|N| \leq 2 \operatorname{diam}(H) \cdot|N| \leq 4 \operatorname{rad}(H) \cdot|N|$ but since $k \geq 9$ and $\operatorname{rad}(H)>2$ (as $k \geq 9$ ) this is a contradiction.

Using the previous two lemmas we can show the following.

Lemma 14 If $G$ is an equilibrium graph for $\alpha>19 n$ then for every vertex $v$ in $H$ there is a vertex $w \in N_{11}(v)$ with $\operatorname{deg}_{H}(w) \geq 3$.

Proof Let $\{u, v\}$ be an arbitrary edge in $H$ and assume without loss of generality that $u$ bought the edge. Let $C$ be a cycle containing $\{u, v\}$ and note that by Lemma 11 it has at least 22 vertices. Denote the vertices after $u$ and $v$ (in that order) in $C$ by $x_{0}, x_{1}, x_{2}, \ldots$ We distinguish two cases. Assume first that there is an $1 \leq i \leq 9$ that buys the edge $\left\{x_{i-1}, x_{i}\right\}$. As we have girth at least 22 (Lemma 11) $u, v, x_{0}, \ldots, x_{i}$ is a shortest $u$ - $x_{i}$-path and therefore, by Lemma 12, either $\operatorname{deg}_{H}\left(x_{i-1}\right) \geq 3$ or $\operatorname{deg}_{H}(v) \geq 3$. Hence since $v, x_{i-1} \in N_{9}(v) \subseteq N_{10}(u)$ the claim is true for that case. Assume now that there is no such $x_{i}$. But then we have a path $x_{0}, x_{1}, \ldots, x_{9}$ of 
length 9 where one vertex buys the edge to the next one. Thus, by Lemma 13, the vertices of the path cannot have all degree 2 in $H$, and the lemma follows.

Now, quite in the same way as for MAXGAME, we can prove the claims that show the main result of the section.

Corollary 5 If $G$ is an equilibrium graph for $\alpha>19$ n then $\operatorname{deg}(H) \geq 2+\frac{1}{34}$.

Proof The proof is completely analog to the proof of Corollary 3.

The following lemma as well as its proof is completely analog to Lemma 10 for MAXGAME and gives us a corresponding upper bound for $\operatorname{deg}(H)$. For completeness we also list the entire proof here.

Lemma 15 If $G$ is an equilibrium graph for $\alpha>n$ then $\operatorname{deg}(H) \leq 2+\frac{8 n}{\alpha-n}$.

Proof Consider a breadth-first search tree $T$ of $G$ rooted in a vertex $v_{0} \in V$ having the minimum usage cost among all vertices. Recall that the usage cost of vertex $v$ in graph $G$ is $\sum_{u \in V} d_{G}(v, u)$, which we denote by $U_{G}(v)$. Let $\tilde{T}:=T \cap H$. Note that $\tilde{T}$ is a spanning tree of $H$. Then $\operatorname{deg}(H)=\frac{2|E(\tilde{T})|+2|E(H) \backslash E(\tilde{T})|}{|V(\tilde{T})|} \leq 2+\frac{2|E(H) \backslash E(\tilde{T})|}{|V(\tilde{T})|}$, and hence we have to bound $|E(H) \backslash E(\tilde{T})|$ (the number of edges outside $\tilde{T}$ ). To do that, we consider vertices of $H$ that buy an edge in $E(H) \backslash E(\tilde{T})$. Let us call such a vertex a shopping vertex. First observe that every shopping vertex $u$ buys exactly one edge in $E(H) \backslash E(\tilde{T})$, as otherwise $u$ could opt not to buy these edges and buy one edge to $v_{0}$ instead, thus saving at least $\alpha$ on creation cost, and having usage cost at most $U_{G}\left(v_{0}\right)+n \leq U_{G}(u)+n$, which (for $\alpha>n$ ) would be an improvement, a contradiction. This immediately shows that there are at most $|V(\tilde{T})|$ edges in $E(H) \backslash$ $E(\tilde{T})$. To get a better bound, we bound the number of shopping vertices. We show that the distance in $\tilde{T}$ between any two shopping vertices is at least $\frac{\alpha-n}{2 n}$. The upper bound on the number of shopping vertices follows: Assign every node $v$ of $H$ to the closest shopping vertex (closest according to the distance in $\tilde{T}$; breaking ties arbitrarily); Observe that this assignment forms a partition of $H$ (and that every part contains exactly one shopping vertex); As the distance in $\tilde{T}$ between any two shopping vertices is at least $\frac{\alpha-n}{2 n}$, the size of every part is at least $\frac{\alpha-n}{4 n}$. Thus, there are at most $\frac{4 n|V(\tilde{T})|}{\alpha-n}$ shopping vertices and thus at most that many edges in $E(H) \backslash E(\tilde{T})$; The desired bound $\operatorname{deg}(H) \leq 2+\frac{8 n}{\alpha-n}$ now easily follows.

We are left to prove that the distance in $\tilde{T}$ between any two shopping vertices is at least $\frac{\alpha-n}{2 n}$. Assume for contradiction that there are two shopping vertices $u_{1} \neq u_{2}$ for which $d_{\tilde{T}}\left(u_{1}, u_{2}\right)<\frac{\alpha-n}{2 n}$. Let $u_{1}=x_{1}, x_{2}, \ldots, x_{k}=u_{2}$ be the shortest $u_{1}-u_{2}$-path in $\tilde{T}$ and let us call it $P$. Let $\left\{u_{1}, v_{1}\right\}$ and $\left\{u_{2}, v_{2}\right\}$ be the edges that $u_{1}$ and $u_{2}$ buy in $E(H) \backslash E(\tilde{T})$. Observe that $v_{1}$ and $v_{2}$ are not descendant of any vertex $x_{i}$, $i=1, \ldots, k$, in $P$; If $v_{j}, j=1,2$, is descendant of $x_{i}$, then the $v_{j}-x_{i}$-path in $\tilde{T}$, the $x_{i}$ $u_{j}$-path in $\tilde{T}$, and the edge $\left\{u_{j}, v_{j}\right\}$ form a cycle of length at most $2\left(d_{\tilde{T}}\left(u_{1}, u_{2}\right)+1\right)<$ $\frac{\alpha}{n}+1$ which contradicts Lemma 11 . In particular, $v_{j}$ is not part of $P$, and therefore 
$x_{0}=v_{1}, x_{1}, \ldots, x_{k}, x_{k+1}=v_{2}$ is a path in $H$. Also by Lemma $11, u_{j}, j=1,2$, has distance at least $\frac{\alpha-n}{2 n}$ from $v_{0}$, and therefore $v_{0}$ is not in $P$. Now, since $x_{1}$ buys $\left\{x_{0}, x_{1}\right\}$ and $x_{k}$ buys $\left\{x_{k}, x_{k+1}\right\}$, there has to be $1 \leq i^{*} \leq k$ such that $x_{i}$ buys both $\left\{x_{i^{*}-1}, x_{i^{*}}\right\}$ and $\left\{x_{i^{*}}, x_{i^{*}+1}\right\}$. Consider the following modification of $x_{i^{*}}$ 's strategy: Buy edge $\left\{x_{i^{*}}, v_{0}\right\}$ instead of edges $\left\{x_{i^{*}-1}, x_{i^{*}}\right\}$ and $\left\{x_{i^{*}}, x_{i^{*}+1}\right\}$. In this new strategy, $x_{i}$ decreases its creation cost by $\alpha$. We now show that $x_{i *}$ 's new usage cost is $U_{\text {new }}\left(x_{i *}\right)<U_{G}\left(x_{i *}\right)+\alpha$ thus implying that the new strategy improves $x_{i}$ 's (total) cost, a contradiction.

First note that $U_{\text {new }}\left(x_{i^{*}}\right) \leq n+U_{\text {new }}\left(v_{0}\right)$ (where the subscript "new" always corresponds to the situation in a graph where $x_{i}$ is using the modified strategy). To bound $U_{\text {new }}\left(v_{0}\right)$ we note that only the vertices in $P$ and their descendants in $T$ can have increased distance to $v_{0}$ by the strategy change. Let $y$ be one of these vertices with possibly increased distance and let $1 \leq j \leq k$ be such that $x_{j}$ is the closest ancestor of $y$, i.e, an ancestor with $d_{G}\left(x_{j}, y\right)=\min _{x \in P} d_{G}(x, y)$. If $j=i^{*}$ it is easy to see that $d_{\text {new }}\left(v_{0}, y\right) \leq d_{G}\left(v_{0}, y\right)$ and therefore for such a vertex $y$ there is no increase in usage cost of $v_{0}$. Consider now the case $j \neq i^{*}$ and assume (without loss of generality, as we shall see) that $j<i^{*}$. Then $d_{\text {new }}\left(v_{0}, y\right) \leq$ $d_{\text {new }}\left(v_{0}, x_{0}\right)+d_{\text {new }}\left(x_{0}, x_{j}\right)+d_{\text {new }}\left(x_{j}, y\right)=d_{G}\left(v_{0}, x_{0}\right)+d_{G}\left(x_{0}, x_{j}\right)+d_{G}\left(x_{j}, y\right)$ (since $x_{0}$ is not a descendant of a vertex in $P$ and $x_{0}, \ldots, x_{j}$ is still a path in $G_{\text {new }}$ ), and $d_{G}\left(v_{0}, y\right)=d_{G}\left(v_{0}, x_{j}\right)+d_{G}\left(x_{j}, y\right)$. Then the increase of distance from $v_{0}$ to $y$ is $d_{\text {new }}\left(v_{0}, y\right)-d_{G}\left(v_{0}, y\right)=d_{G}\left(v_{0}, x_{0}\right)+d_{G}\left(x_{0}, x_{j}\right)-d_{G}\left(v_{0}, x_{j}\right) \leq 2 \cdot d_{G}\left(x_{0}, x_{j}\right) \leq$ $2 \cdot d_{G}\left(u_{1}, u_{2}\right) \leq 2 \cdot d_{\tilde{T}}\left(u_{1}, u_{2}\right)<\frac{\alpha-n}{n}$, where the last inequality follows from our assumption $d_{\tilde{T}}\left(u_{1}, u_{2}\right)<\frac{\alpha-n}{2 n}$. As $y$ was chosen arbitrary, we have that the increase of usage cost of $v_{0}$ is less than $n \frac{\alpha-n}{n}=\alpha-n$ and therefore $U_{\text {new }}\left(v_{0}\right)<U_{G}\left(v_{0}\right)+\alpha-n$, which shows that $U_{\text {new }}\left(x_{i}\right)<U_{G}\left(x_{i^{*}}\right)+\alpha$, as desired.

We are now ready to state the main structural result of the section.

Theorem 7 For $\alpha>273 n$ every equilibrium graph is a tree.

Proof If $G$ is a non-tree equilibrium for $\alpha>273 n$ and $H$ a block in $G$ with $|H| \geq 3$ then we have by Lemma 15 that $\operatorname{deg}(H) \leq 2+\frac{8 n}{\alpha-n}<2+\frac{1}{34}$, which contradicts Corollary 5 stating that $\operatorname{deg}(H) \geq 2+\frac{1}{34}$.

We note that this bound is asymptotically tight, as Albers et al. showed in [1] that for $\alpha<n / 2$ there exist equilibrium graphs which are not trees. Actually, there are known constructions of non-tree equilibrium graphs for every value of $\alpha<n$ [17]. To prove the main result of the section, we recall the following theorem.

Theorem 8 [8] The cost of an equilibrium graph that is a tree is less than 5 times the cost of a social optimum.

Theorems 7 and 8 immediately give the following corollary.

Corollary 6 For $\alpha>273 n$ the price of anarchy is smaller than 5. 


\section{Conclusions}

In this article we have considered the two most prominent versions of the (nowadays) broad class of network creation games-the SuMGAME and the MAXGAME. We have improved the upper bounds on the price of anarchy for a large range of the edge prices $\alpha$. As a main result, we have shown that the price of anarchy is upper-bounded by a constant for every SUMGAME where $\alpha>273 n$, and by a constant for every MAXGAME where $\alpha>129$. To prove these bounds, we have shown that for these ranges of $\alpha$, only trees can appear as Nash equilibria. Obviously, the most natural open problem asks whether the price of anarchy is upper-bounded by a constant for all values of $\alpha$. There are other interesting open questions. Perhaps one of the most imminent one asks for an upper bound on the diameter of an equilibrium graph. Until now, only equilibrium graphs with logarithmic diameter are known.

Acknowledgements This work has been partially supported by the Swiss National Science Foundation (SNF) under the grant number 200021_143323/1.

\section{References}

1. Albers, S., Eilts, S., Even-Dar, E., Mansour, Y., Roditty, L.: On Nash equilibria for a network creation game. In: Proc. 17th Annual ACM-SIAM Symposium on Discrete Algorithms (SODA), pp. 89-98. ACM, New York (2006). doi:10.1145/1109557.1109568

2. Alon, N., Demaine, E.D., Tom Leighton, M.H.: Basic network creation games. In: Proc. 22nd ACM Symposium on Parallelism in Algorithms and Architectures (SPAA), pp. 106-113. ACM, New York (2010). doi: $10.1145 / 1810479.1810502$

3. Bilò, D., Gualà, L., Leucci, S., Proietti, G.: The max-distance network creation game on general host graphs. In: Proc. 8th International Workshop on Internet and Network Economics (WINE), pp. 392405 (2012). doi:10.1007/978-3-642-35311-6_29

4. Bilò, D., Gualà, L., Proietti, G.: Bounded-distance network creation games. In: Proc. 8th International Workshop on Internet and Network Economics (WINE), pp. 72-85 (2012). doi:10.1007/978-3642-35311-6_6

5. Brautbar, M., Kearns, M.: A clustering coefficient network formation game. In: Proc. Fourth International Symposium on Algorithmic Game Theory (SAGT), pp. 224-235 (2011). doi:10.1007/ 978-3-642-24829-0_21

6. Demaine, E.D., Hajiaghayi, M., Mahini, H., Zadimoghaddam, M.: The price of anarchy in network creation games. ACM Trans. Algorithms 8(2), 1-13 (2012). doi:10.1145/2151171.2151176

7. Ehsani, S., Fazli, M., Mehrabian, A., Sadeghian Sadeghabad, S., Safari, M., Saghafian, M., ShokatFadaee, S.: On a bounded budget network creation game. In: Proc. 23rd ACM Symposium on Parallelism in Algorithms and Architectures (SPAA), pp. 207-214 (2011). doi:10.1145/1989493.1989523

8. Fabrikant, A., Luthra, A., Maneva, E., Papadimitriou, C.H., Shenker, S.: On a network creation game. In: Proc. 22nd Annual Symposium on Principles of Distributed Computing (PODC), pp. 347-351. ACM, New York (2003). doi:10.1145/872035.872088

9. Garey, M.R., Johnson, D.: Computers and Intractability: a Guide to the Theory of NP-completeness. Freeman, San Francisco (1979)

10. Jackson, M.O.: Social and Economic Networks. Princeton University Press, Princeton (2008)

11. Jackson, M.O., Wolinski, A.: A strategic model of social and economic networks. J. Econ. Theory 71(1), 44-74 (1996). doi:10.1006/jeth.1996.0108

12. Kawald, B., Lenzner, P.: On dynamics in selfish network creation. CoRR (2012). arXiv:1212.4797 [cs.GT]

13. Lenzner, P.: On dynamics in basic network creation games. In: Proc. Fourth International Symposium on Algorithmic Game Theory (SAGT), pp. 254-265 (2011). doi:10.1007/978-3-642-24829-0_23

14. Lenzner, P.: Greedy selfish network creation. In: Proc. Eighth International Workshop on Internet and Network Economics (WINE), pp. 142-155 (2012). doi:10.1007/978-3-642-35311-6_11 
15. Lin, H.: On the price of anarchy of a network creation game (2003). Final class-project

16. Mihalák, M., Schlegel, J.C.: Asymmetric swap-equilibrium: a unifying equilibrium concept for network creation games. In: Proc. 37th International Symposium on Mathematical Foundations of Computer Science (MFCS), pp. 693-704 (2012). doi:10.1007/978-3-642-32589-2_60

17. Müller, D.: About constricted cycles in Nash equilibria of the local connection game. Semester thesis, ETH, Zurich (2012)

18. Tardos, E., Wexler, T.: Network formation games. In: Nisan, N., Rougarden, T., Tardos, É., Vazirani, V. (eds.) Algorithmic Game Theory. Cambridge University Press, Cambridge (2007) 